\title{
Factors associated with pulmonary toxicity after myeloablative conditioning using fractionated total body irradiation
}

Hwa Kyung Byun, MD', Hong In Yoon, MD, PhD¹, Jaeho Cho, MD, PhD¹, Hyun Ju Kim, MD¹, Yoo Hong Min, MD, PhD², Chuhl Joo Lyu, MD, PhD³, June-Won Cheong, MD, PhD², Jin Seok Kim, MD, PhD², Hyo Sun Kim, MD³, Soo-Jeong Kim, MD², Andrew Jihoon Yang, MD¹, Byung Min Lee, MD', Won Hee Lee, MD', Joongyo Lee, MD', Ki Jung Ahn, MD', Chang-Ok Suh, MD, PhD ${ }^{1}$

'Department of Radiation Oncology, Yonsei Cancer Center, Yonsei University College of Medicine, Seoul;

${ }^{2}$ Division of Hematology, Department of Internal Medicine, Severance Hospital, Yonsei University College of Medicine, Seoul;

${ }^{3}$ Division of Pediatric Hemato-oncology, Department of Pediatrics, Yonsei University Health System,

Yonsei University College of Medicine, Seoul; ${ }^{4}$ Department of Radiation Oncology, Inje University Busan Paik Hospital,

Inje University College of Medicine, Busan, Korea

Purpose: Pulmonary toxicities, including infectious pneumonia (IP) and idiopathic pneumonia syndrome (IPS), are serious side effects of total body irradiation (TBI) used for myeloablative conditioning. This study aimed to evaluate clinical factors associated with IP and IPS following TBI.

Materials and Methods: Fifty-eight patients with hematologic malignancies who underwent TBI before allogeneic hematopoietic stem cell transplantation between 2005 and 2014 were reviewed. Most patients (91\%) received 12 Gy in 1.5 Gy fractions twice a day. Pulmonary toxicities were diagnosed based on either radiographic evidence or reduced pulmonary function, and were subdivided into IP and IPS based on the presence or absence of concurrent infection.

Results: Pulmonary toxicities developed in 36 patients (62\%); $16(28 \%)$ had IP and 20 (34\%) had IPS. IP was significantly associated with increased treatment-related mortality $(p=0.028)$ and decreased survival $(p=0.039)$. Multivariate analysis revealed that the risk of developing IPS was significantly higher in patients who received stem cells from a matched unrelated donor than from a matched sibling donor $(p=0.021$; hazard ratio $[H R]=12.67 ; 95 \%$ confidence interval $[\mathrm{Cl}]$, 1.46-110.30). Combining other conditioning agents with cyclophosphamide produced a higher tendency to develop IP ( $p=0.064 ; \mathrm{HR}=6.19 ; 95 \% \mathrm{Cl}, 0.90-42.56)$. Conclusion: IP and IPS involve different risk factors and distinct pathogeneses that should be considered when planning treatments before and after TBI.

Keywords: Total body irradiation, Infectious pneumonia, Idiopathic pneumonia syndrome, Stem cell transplantation

Received 29 May 2017, Revised 14 August 2017, Accepted 16 August 2017.

Correspondence: Hong In Yoon, MD, PhD, Department of Radiation Oncology, Yonsei Cancer Center, Yonsei University College of Medicine, 50-1 Yonsei-ro, Seodaemun-gu, Seoul, 03722, Korea. Tel: +82-2-2228-8110, Fax: +82-2-2227-7823, E-mail: YHI0225@yuhs.ac

(c) This is an Open Access article distributed under the terms of the Creative Commons Attribution Non-Commercial License (http://creativecommons.org/ licenses/by-nc/4.0/) which permits unrestricted non-commercial use, distribution, and reproduction in any medium, provided the original work is properly cited.

www.e-roj.org 


\section{Introduction}

Total body irradiation (TBI) is a radiotherapy technique frequently used as a component of hematopoietic stem cell transplantation (HSCT). TBI used in conjunction with chemotherapeutic agents has proven to be useful for eradicating residual malignant or genetically abnormal cells. It is also used for immunosuppression prior to HSCT, and its accessibility to so-called sanctuary sites is a major advantage $[1,2]$. However, various acute and late side effects that include gastrointestinal symptoms, cataract, hepatic veno-occlusive disease, and renal complications can develop after TBI-based HSCT, and can sometimes be life-threatening $[3,4]$. Among the serious side effects related to TBI-based HSCT, pulmonary toxicity was the single major reason for transplantationassociated deaths, with an incidence rate of up to $62 \%$ and a mortality rate of up to $65 \%$ in early studies $[5,6]$

Pulmonary toxicities following HSCT can result from infectious and/or noninfectious etiologies. Infectious etiologies typically include bacterial, viral, or fungal pneumonia; noninfectious etiologies are best characterized by the presumed site of primary tissue injury. In 1993, the term idiopathic pneumonia syndrome (IPS) was proposed to describe widespread alveolar injury in the absence of active lower respiratory tract infection after bone marrow transplantation [7]. The two types of pulmonary toxicities, infectious pneumonia (IP) and IPS, are considered distinct disease entities because they have different etiologies, treatments, and prognoses. Various clinical parameters have been studied to identify factors associated with pulmonary toxicity after TBI-based HSCT. However, the factors that influence the development of IP and IPS after TBI-based stem cell transplantation remain controversial [8-10]. Given the distinct pathogeneses of the two types of pneumonia, we hypothesized that IP and IPS have their own unique risk factors. A better understanding of such risk factors related to IP and IPS is important because even though several investigators have attempted to reduce pulmonary toxicities by modifying radiation-related factors such as dose-rate or total dose, these fatal toxicities have not been completely prevented and still result in high mortality rates $[10,11]$. Our aim was to identify the clinicpathological factors associated with IP and IPS that develop after fractionated TBI administered as a part of a myeloablative conditioning regimen before HSCT.

\section{Materials and Methods}

\section{Patient population}

We retrospectively reviewed 69 patients with hematologic malignancies who received TBI as part of a conditioning regimen before allogeneic stem cell transplantation between January 2005 and December 2014 at Yonsei Cancer Center. According to our institution's treatment protocol, TBI-based myeloablative conditioning was performed only for patients with high-risk, very high-risk, or refractory hematologic malignancies. Patients who received autologous stem cell transplantation $(n=6)$ or nonmyeloablative TBI $(n=5)$ were excluded; those who underwent stem cell transplantation without TBI were not analyzed. Fifty-eight patients were ultimately eligible for this analysis.

\section{Stem cell transplantation procedure}

All patients underwent a standard pre-HSCT evaluation, including a pulmonary function test, complete blood count, and chemistry profile. If cardiopulmonary, hepatic, and renal functions remained adequate, TBI-based conditioning regimens were initiated before the HSCT. Chemotherapy conditioning prior to TBI was generally performed using high-dose cyclophosphamide (51 of 58 cases). Variations in the regimen were influenced by underlying diseases, risk of complications, and patient preference. TBI was performed for 4 days from day -8 to day -5 , after which patients received high-dose cyclophosphamide $(60 \mathrm{mg} / \mathrm{kg}$ in 2 doses on days -3 and -2 ). Intrathecal methotrexate was administered on day -3 for central nervous system prophylaxis. From the day of initiation of $\mathrm{TBI}$, micafungin, acyclovir, and trimethoprimsulfamethoxazole were used for antifungal prophylaxis, antiviral prophylaxis, and prophylaxis against Pneumocystis jirovecii, respectively.

\section{TBI technique}

Generally, a simulation was performed in the treatment position 1 week before the initiation of TBI. Thickness measurements were obtained for the patients at the prescription point and at other points of interest for dose calculations and determination of homogeneity, such as measurements of the head, neck, shoulder, lung, mediastinum, xiphoid, pelvis, thigh, knee, calf, and ankle. An individualized lead compensator was made to homogenize the wholebody dose to within $\pm 5 \%$ in all patients. Calculations were performed by a qualified medical physicist to achieve the prescribed dose, dose homogeneity, and any other relevant 
dose points. Patients were placed in the supine position, with whole-body vacuum immobilization against the far wall of the linear accelerator room to achieve maximum source-to-surface distance and field size in order to encompass the entire body. If the patients were too tall to fit into the field-range, a bentknee posture was adopted; otherwise, the patients lay with their legs straightened. Treatment was administered using a linear accelerator with $10 \mathrm{MV}$ photons, bilateral parallel opposing fields, and an extended source-to-axis distance (SAD = 390 $\mathrm{cm}$ ). Thermoluminescence dosimetry was applied to the head, neck, chest (surface and lateral), and pelvis, as well as to the medial sides of the thigh, knee, and malleolus. Total TBI doses ranged from 8 to $12 \mathrm{~Gy}$; most of the patients (91\%) received $12 \mathrm{~Gy}$. All patients received fractionated TBI in mostly $1.5 \mathrm{~Gy}$ fractions (94.8\%) twice a day. Both the lungs were intended to receive the prescribed radiation dose. We did not perform lung dose reduction. Both arms of the patient were laid down along the sides of the body such that the shadow of the arms would partially cover the lungs. To compensate the low density of the lung, lead compensators were designed considering the thickness of an arm. The dose rate was between 7 and 19 cGyl min. In November 2011, the specific TBI guideline used by our treatment team was partially modified, and the dose rate was increased from 5-10 to 12-20 cGy/min. The dose rate was then subtly adjusted according to each patient's condition; comorbidities (such as a prior history of pneumonia) were always considered.

\section{Follow-up, assessment, and definition of pulmonary toxicity}

Patients were hospitalized for at least for 1 month postHSCT until their acute toxicity was managed sufficiently. After discharge, regular examinations including chest radiography, physical examination, bone marrow aspiration, and a complete blood count were performed at regular visits that occurred with intervals of 1-2 months during the first year, 3 months during the second year, and 6 months thereafter. Baseline pulmonary function tests were obtained within 1 month before TBI. The diffusion capacity of carbon dioxide was determined using a carbon monoxide single-breath technique with correction for hemoglobin concentration. Patients with clinical pulmonary symptoms, such as dyspnea/cough or any indication of respiratory symptoms, were defined as having pulmonary toxicity only if they showed radiographic evidence or reduced pulmonary function; this was to reduce the falsepositive rate. Pulmonary toxicity was further subdivided based on the presence or absence of concurrent infection. IPS was defined as pulmonary toxicity with no signs of infection, whereas IP was defined as pulmonary toxicity with signs of infection in the blood, bronchoalveolar lavage, or other specimens. Cultures, serological tests, and/or polymerase chain reaction were used to identify IP-causing pathogens in the specimens.

\section{Survival outcomes}

The actuarial overall survival (OS) was calculated as the duration between the end of TBI and either death or the last follow-up date. Treatment-related mortality (TRM) was defined as any death from any cause other than relapse or refractory disease, as described in previous studies $[12,13]$. Acute graftversus-host disease (GVHD) was graded using the Glucksberg grading system, where each of the three organs (skin, gastrointestinal tract, and liver) is staged from 0 to 4 ; staging includes objective assessment of organ function and subjective assessment of performance status. According to the grading system, Grades 1-2 included cases with mild-to-moderate skin changes, no or mild gastrointestinal involvement, and no or mild decreases in clinical performance; Grades 3-4 included more serious diseases [14].

\section{Statistical analysis}

The relationship between pulmonary toxicity and clinical and therapeutic factors such as age, sex, disease types, dose rate, total dose, acute GVHD, use of methotrexate for prophylaxis against acute GVHD, transfusion cell type, number of complete remissions, donor type, conditioning chemotherapy regimens, and pre-transplantation pulmonary function were further investigated. The associations of IP and IPS with other clinical variables on univariate and multivariate analyses were examined using binary logistic regression. Because it was unclear whether the clinical characteristics of the patients who died early without developing pulmonary toxicity affected the occurrence of the event, patients who died within 6 months after TBI ( $n=9$ ) were excluded from the univariate and multivariate logistic regression analyses. This exclusion was regarding the typical onset of pulmonary toxicities of 1-6 months, and to reduce the potential underestimation problem. Multivariate analysis of IP and IPS was conducted by including factors with a p-value $<0.10$ on univariate analysis; a backward variable selection method was used with an alpha level of removal of 0.1. A two-sided p-value of $<0.05$ was considered significant. OS was estimated using the KaplanMeier method, and a log-rank test was used to assess the difference between patients with and without pulmonary 
Table 1. Patients' characteristics $(n=58)$

\begin{tabular}{|c|c|}
\hline Characteristic & Value \\
\hline \multicolumn{2}{|l|}{ Age (yr) } \\
\hline$<20$ & $38(65.5)$ \\
\hline$\geq 20$ & $20(34.5)$ \\
\hline \multicolumn{2}{|l|}{ Sex } \\
\hline Female & $22(37.9)$ \\
\hline Male & $36(62.1)$ \\
\hline \multicolumn{2}{|l|}{ Disease } \\
\hline ALL & $43(74.1)$ \\
\hline $\mathrm{AML}$ & 8 (13.8) \\
\hline Other & $7(12.1)$ \\
\hline \multicolumn{2}{|l|}{ Total dose } \\
\hline 8 Gy (2.0 Gy × 4 fx, once daily) & $1(1.7)$ \\
\hline 9 Gy (1.5 Gy × 6 fx, twice daily) & $2(3.4)$ \\
\hline 9.6 Gy (1.2 Gy × $8 \mathrm{fx}$, twice daily) & $1(1.7)$ \\
\hline $12 \mathrm{~Gy}$ (2.0 Gy × 6 fx, twice daily) & $1(1.7)$ \\
\hline 12 Gy (1.5 Gy × 8 fx, twice daily) & $53(91.4)$ \\
\hline \multicolumn{2}{|l|}{ Dose rate (cGy/min) } \\
\hline $5-9.99$ & $20(34.5)$ \\
\hline $10-14.99$ & $20(34.5)$ \\
\hline$\geq 15$ & $18(31.0)$ \\
\hline \multicolumn{2}{|l|}{ Donor type } \\
\hline Matched sibling & $12(20.7)$ \\
\hline Matched unrelated & $41(70.7)$ \\
\hline Mismatched related & $5(8.6)$ \\
\hline \multicolumn{2}{|l|}{ Cell type } \\
\hline Bone marrow & $22(37.9)$ \\
\hline Peripheral blood & $33(56.9)$ \\
\hline Cord blood & $3(5.2)$ \\
\hline \multicolumn{2}{|l|}{ Conditioning regimen group } \\
\hline Cy & $42(72.4)$ \\
\hline $\mathrm{Cy}$ +other & $9(15.5)$ \\
\hline Other & $7(12.1)$ \\
\hline \multicolumn{2}{|l|}{ Acute GVHD prophylaxis } \\
\hline No MTX & $14(24.1)$ \\
\hline Yes MTX & $44(75.9)$ \\
\hline \multicolumn{2}{|l|}{ (If leukemia) CR number } \\
\hline Refractory & $3(5.9)$ \\
\hline CR1 & $31(60.8)$ \\
\hline$\geq \mathrm{CR} 2$ & $17(33.3)$ \\
\hline preFEV1 & $87.9(50.0-127.0)$ \\
\hline preFVC & $86.1(56.1-131.0)$ \\
\hline preDLCO & $66.0(51.0-80.0)$ \\
\hline \multicolumn{2}{|l|}{ Smoking history } \\
\hline No & $53(91.4)$ \\
\hline Yes & $5(8.6)$ \\
\hline \multicolumn{2}{|l|}{ Pre-transplant pulmonary comorbidity } \\
\hline No & $53(91.4)$ \\
\hline Yes & $5(8.6)$ \\
\hline
\end{tabular}

Values are presented as number (\%) or median (range).

$A L L$, acute lymphoblastic leukemia; $A M L$, acute myeloid leukemia; Cy, cyclophosphamide; GVHD, graft-versus-host disease; MTX, methotrexate; $C R$, complete response; FEV1, forced expiratory volume in 1 second; FVC, forced vital capacity; DLCO, diffusion capacity of carbon monoxide.
Table 2. Causes of death $(n=58)$

\begin{tabular}{lc}
\hline \multicolumn{1}{c}{ Variable } & No. (\%) \\
\hline Total as of July 2016 & $37(63.8)$ \\
Relapse or refractory disease related & $16(27.6)$ \\
Treatment related & $21(36.2)$ \\
Infectious pneumonia & $7(12.1)$ \\
Idiopathic pneumonia syndrome & $6(10.3)$ \\
Acute graft-versus-host disease & $3(5.2)$ \\
Acute renal failure & $1(1.7)$ \\
Hepatic veno-occlusive disease & $1(1.7)$ \\
Secondary malignancy & $1(1.7)$ \\
Sedation & $1(1.7)$ \\
Other infection & $1(1.7)$ \\
\hline
\end{tabular}

toxicities. TRM and cumulative incidences of IPS and IP were estimated with competing risks analyses; TRM \& relapse and IPS \& IP were considered as competing risks, respectively. The comparisons between subgroups were performed using the Gray's test $[1,2]$. We used the R package 'cmprsk' for the analyses (v2.2-7, https://CRAN.R-project.org/package=cmprsk) with R software (https://www.R-project.org/). All other analyses were performed using SPSS ver. 20.0 (IBM, Armonk, NY, USA).

\section{Results}

\section{Patients and treatment characteristics}

Fifty-eight patients were eligible for the study. The median follow-up was 15 months (range, 13 days to 132 months). Patient characteristics and treatment details are listed in Table 1. Most patients were treated for acute lymphoblastic leukemia (74.1\%) or myelogenous leukemia (13.8\%). Five patients had Iymphoma (8.6\%), 1 had neuroblastoma (1.7\%), and 1 had Ewing sarcoma (1.7\%). The conditioning regimens used with $\mathrm{TBI}$ were cyclophosphamide alone and cyclophosphamide plus other agents in 42 and 9 patients, respectively; other agents included etoposide $(n=4)$, etoposide plus imatinib $(n=1)$, fludarabine $(n=2)$, cytarabine $(n=1)$, and antithymocyte globulin $(n=1)$. Pre-transplantation pulmonary comorbidities developed in 5 patients before the initiation of the preparative regimen. These included atypical pneumonia $(n=1)$, pulmonary tuberculosis $(n=1)$, EBV pneumonitis ( $n=1)$, fungal pneumonia $(n=1)$, and pneumonia of unknown etiology $(n=1)$, all of which were well controlled or completely treated at the time of transplantation. Stem cells were derived from a matched sibling (20.7\%), a matched unrelated $(70.7 \%)$, or a mismatched related $(8.6 \%)$ donor. Stem 
A

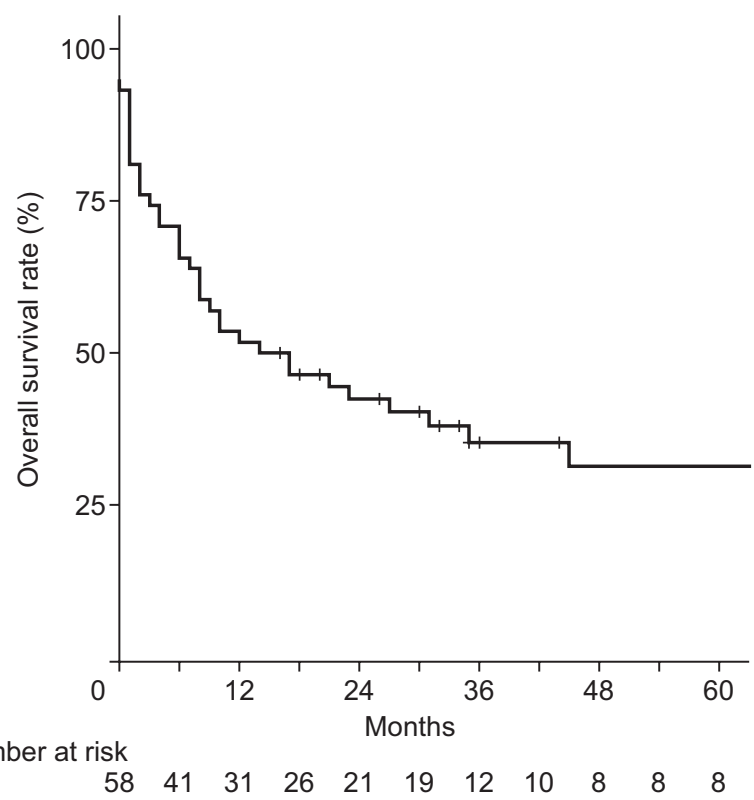

B

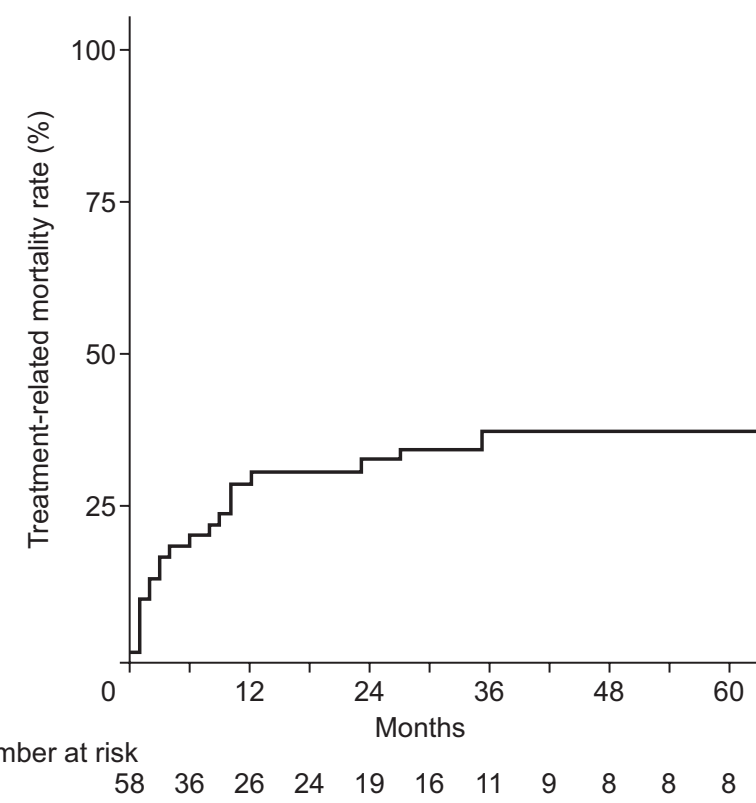

Fig. 1. (A) Probabilities of overall survival in 58 patients. (B) Probabilities of treatment-related mortality in 58 patients.

cells included mobilized peripheral blood stem cells in 56.9\%, bone marrow in $37.9 \%$, and cord blood in $5.2 \%$ of patients. Post-transplant graft-versus-host prophylaxis included the use of methotrexate in $75.9 \%$ of patients.

\section{Treatment outcomes associated with pulmonary toxicities} In all 58 patients, the 1-, 2-, and 3-year OS rates were 53.4\%, $42.4 \%$, and $35.2 \%$, respectively (Fig. 1A). The 1-, 2-, and 3 -year TRM rates were $31.0 \%, 33.0 \%$, and $37.7 \%$, respectively (Fig. 1B). The causes of death, including both disease-related death and TRM, are listed in Table 2. Thirty-seven of the 58 patients had died by the time of data analysis, 16 and 21 of disease-related and treatment-related causes, respectively. IP and IPS were the two leading causes of TRM, each accounting for 33.3\% and $28.6 \%$ of all treatment-related deaths, respectively. IP was significantly associated with a decrease in the OS rate and an increase in the TRM rate. The 2-year OS rates in patients with and without IP were $25.0 \%$ and $49.3 \%$, respectively $(p=0.039)$ (Fig. 2A). The 2-year TRM rates in patients with and without IP were $56.3 \%$ and $24.1 \%$, respectively $(p=0.028)$ (Fig. $2 B)$. In contrast, IPS had no significant association with either the OS rate $(p=0.264)$ or the TRM rate $(p=0.669)(F i g .2 C, 2 D)$.

\section{Clinical factors associated with developing pulmonary toxicities}

Overall, pulmonary toxicities developed in 36 patients (62\%); $16(28 \%)$ had a concurrent infection while $20(34 \%)$ exhibited no detectable pathogens. The leading etiology of IP was bacterial $(75 \%)$, followed by fungal $(38 \%)$, and viral $(12.5 \%)$. The median time from HSCT to the onset of pulmonary toxicity was 6 (range, 1 to 31) months in patients with IP and 7 (range, 0 to 26) months in patients with IPS. In all 58 patients, the 1$2-$, and 3-year cumulative incidence rates of IPS were 31.0\%, $33.0 \%$, and $37.7 \%$, respectively, and the $1-, 2-$, and 3 -year cumulative incidence rates of IP were $25.9 \%, 29.7 \%$, and 31.8\%, respectively (Fig. 3).

Clinical factors associated with pulmonary toxicities according to univariate and multivariate analyses are listed in Tables 3 and 4. Among the clinical variables analyzed in the univariate analysis, four factors (donor type, cell type, conditioning regimen, acute GVHD prophylaxis) with a p-value $<0.10$ on univariate analysis are included in the multivariate analysis. On univariate analysis, IP was significantly related to the conditioning regimen (cyclophosphamide plus other vs. cyclophosphamide alone; $p=0.028$; hazard ratio $[\mathrm{HR}]=7.20$; 95\% confidence interval [CI], 1.24-41.94); however, IP just missed the threshold for statistical significance on multivariate analysis (cyclophosphamide plus other vs. cyclophosphamide alone; $p=0.064 ; \mathrm{HR}=6.19 ; 95 \% \mathrm{Cl}, 0.90-42.56)$. Separately, IPS showed a significant association with donor type on univariate analysis (matched unrelated vs. matched sibling; $p=0.021 ; H R=12.67 ; 95 \% C l, 1.46-110.30)$ and cell type (peripheral blood vs. bone marrow; $p=0.031 ; H R=4.09 ; 95 \%$ $\mathrm{Cl}, 1.14-14.66)$. Of these, only the donor type was significantly 
A

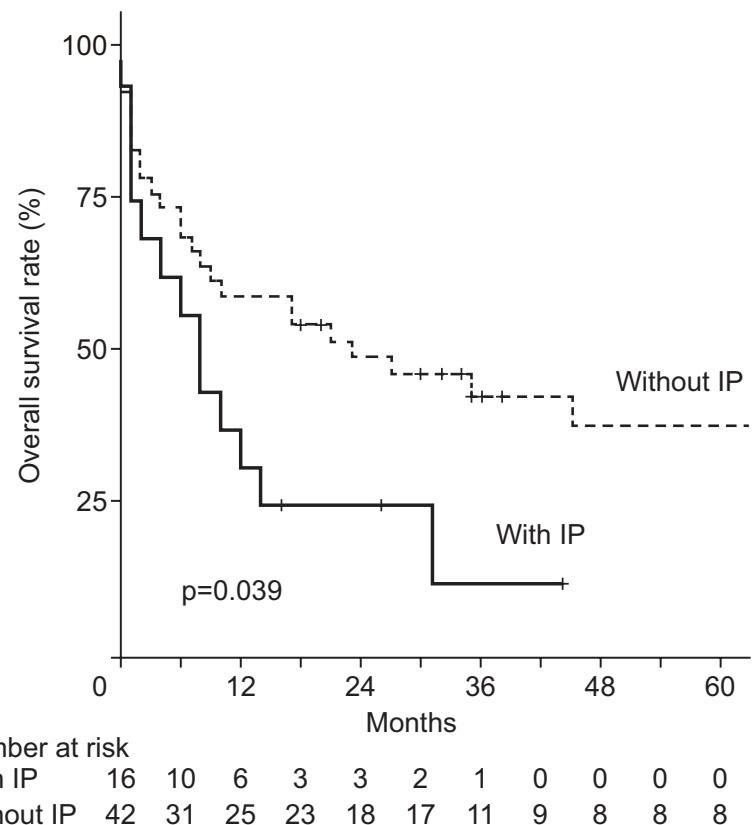

C

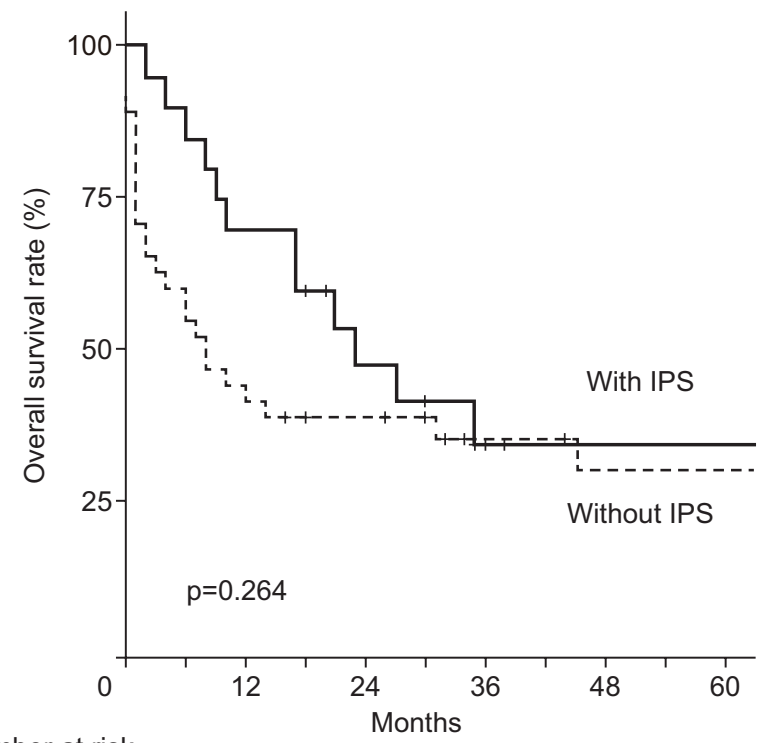

Number at risk

$\begin{array}{llllllllllll}\text { With IPS } & 20 & 15 & 11 & 11 & 7 & 6 & 4 & 2 & 2 & 2 & 2\end{array}$

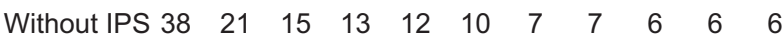

B

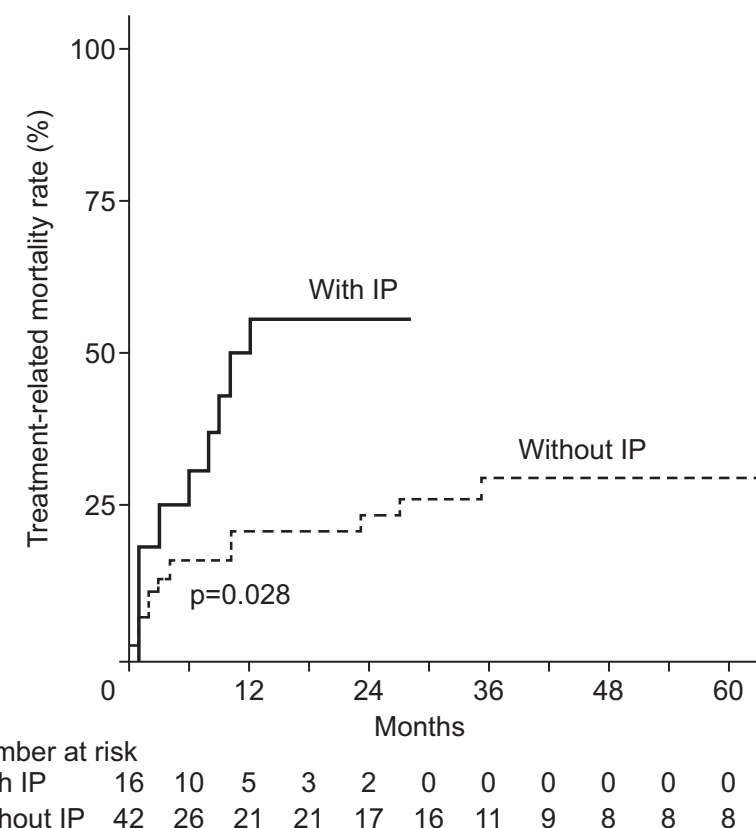

D

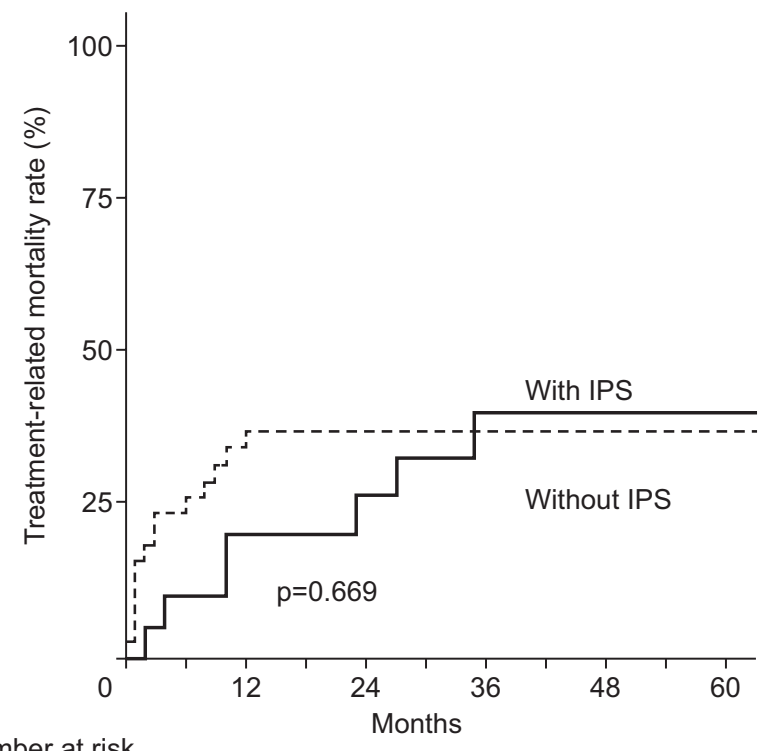

Number at risk

$\begin{array}{llllllllllll}\text { With IPS } & 20 & 18 & 14 & 12 & 8 & 7 & 4 & 2 & 2 & 2 & 2\end{array}$

$\begin{array}{lllllllllll}\text { Without IPS } 38 & 23 & 17 & 14 & 13 & 12 & 8 & 8 & 6 & 6 & 6\end{array}$

Fig. 2. (A) Actuarial survival curves in patients with and without IP. (B) Actuarial incidence of treatment-related mortality in patients with and without IP. (C) Actuarial survival curves in patients with and without IPS. (D) Actuarial incidence of treatment-related mortality in patients with and without IPS. IP, infectious pneumonia; IPS, idiopathic pneumonia syndrome.

associated with IPS on multivariate analysis (matched unrelated vs. matched sibling; $p=0.021 ; \mathrm{HR}=12.67 ; 95 \% \mathrm{Cl}$, 1.46-110.30).

\section{Discussion and Conclusion}

Among the most common causes of treatment-related toxicities are pulmonary complications, which occur in $25 \%-80 \%$ of patients and account for approximately 50\% of transplantation-related deaths $[10,13,15,16]$. Despite 


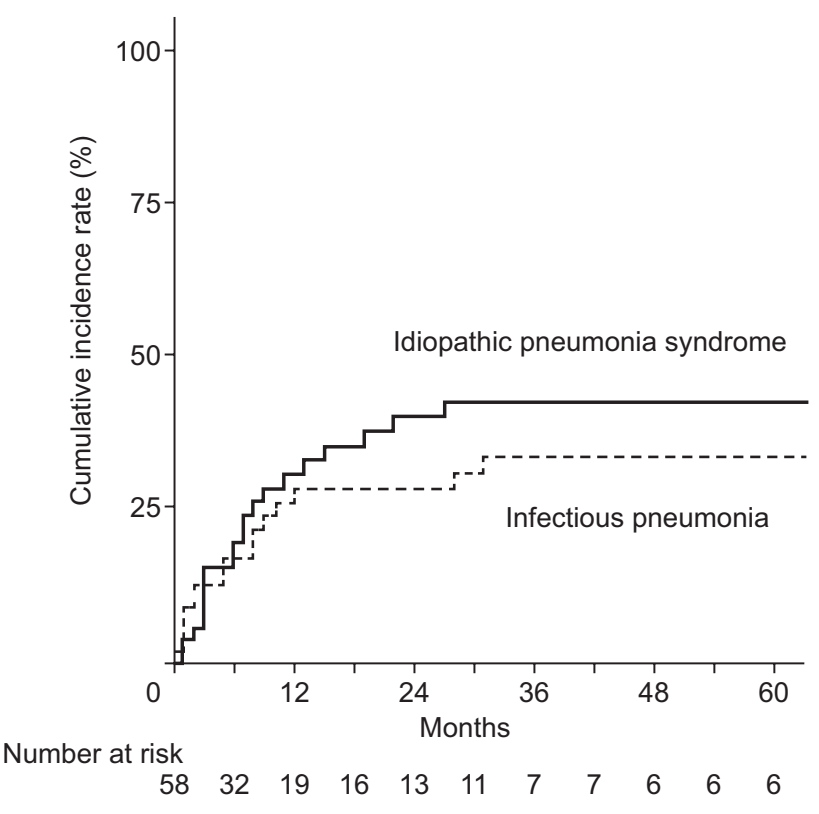

Fig. 3. The cumulative incidence of idiopathic pneumonia syndrome and infectious pneumonia in 58 patients.

the distinct characteristics of IP and IPS, most studies that attempted to identify factors associated with pulmonary toxicity have focused on non-specified types of pulmonary toxicity $[9,16,17]$ or only on IPS $[18,19]$, Moreover, previous studies evaluating pulmonary toxicity after transplantation did not necessarily distinguish between allogeneic and autologous stem cell transplantation $[16,19]$, which makes their findings difficult to compare. The present study has several unique aspects compared with previous studies on pulmonary toxicities after TBI. First, we restricted the inclusion criteria to patients who underwent myeloablative allogeneic HSCT. Second, we applied a single standardized treatment with a uniform total dose, fraction dose, treatment position, and beam delivery system. This homogeneity in patient group and treatment is critical for accurate assessment of the treatmentrelated toxicity because toxicity depends on the intensity and nature of the treatment. Finally, we separately evaluated clinical factors that influence IP and IPS, with the hypothesis that these two major pulmonary complications after TBI-based HSCT have their own unique risk factors.

Donor type and cell type were significantly associated with the development of IPS according to univariate analysis; of these, only the donor type was significant on multivariate regression analysis after adjustment. This indicates that patients who received allografts from a matched unrelated donor had a higher risk of developing IPS than those who received cells from a matched sibling donor, a finding that is consistent with previous reports. Chen et al. [17] found that the incidence of pneumonia was higher in patients with matched unrelated donors than in those with matched related donors, and Zhang et al. [20] found that the risks of GVHD and TRM increase with HLA-mismatch. Donor HLA-mismatch affects the development of post-transplantation pulmonary toxicity; however, only 5 patients received stem cells from an unmatched donor in the present study, 3 of who died within 2 months of TBI. Therefore, the association between an unmatched donor and IPS could not be evaluated because of the small subset of patients and the short survival duration.

On univariate analysis, there was a significant increase in the development of IP when other agents were added to the cyclophosphamide/TBI-based conditioning regimen; and a similar trend was observed on multivariate analysis, although without statistical significance. A myeloablative regimen destroys hematopoietic cells in the bone marrow and causes profound neutropenia and immunodeficiency; therefore, opportunistic infections are major complications after myeloablative HSCT [21]. Several studies have revealed that the increased intensity of a conditioning regimen is associated with post-transplantation infections [22-24]. A previous study suggested that the number of prior chemotherapy regimens is an important risk factor for severe pulmonary toxicity [9]. We suggest that the use of additional regimens with the cyclophosphamide/TBI-based myeloablative conditioning regimen may increase the risk of post-transplantation IP.

The relationship between radiation-related factors and the development of pulmonary toxicity has been strongly debated. Because various radiation therapy techniques are employed, it is difficult to draw definitive conclusions from the available studies. Fractionated TBI for myeloablative HSCT, typically with a total dose of 12-15 Gy delivered in 8-12 fractions, has been more commonly accepted in contemporary practice than single-fraction TBI [25]. All patients in the present study received fractionated $\mathrm{TB}$, and more than 90\% received a total dose of $12 \mathrm{~Gy}$ in $1.5 \mathrm{~Gy}$ fractions twice a day. One of the remaining TBI-related issues is whether the dose rate in modern fractionated TBI affects pulmonary toxicity. Although some studies have found that the dose rate is important $[10,26]$, others argue to the contrary $[27,28]$. In 2011, our institution decided to increase the dose rate from 30\% to 50\% while ensuring that it did not exceed $20 \mathrm{cGy} / \mathrm{min}$. The new dose rate was adopted after considering the efficacy of the treatment, patient convenience, practice guidelines at the time [25], and previously reported data that suggested that the dose rate did 
Table 3. Univariate analysis of factors associated with infectious pneumonia and idiopathic pneumonia syndrome

\begin{tabular}{|c|c|c|c|c|c|c|}
\hline \multirow{2}{*}{ Variable } & \multicolumn{3}{|c|}{ Infectious pneumonia } & \multicolumn{3}{|c|}{ Idiopathic pneumonia syndrome } \\
\hline & $\mathrm{OR}$ & $95 \% \mathrm{Cl}$ & p-value* & OR & $95 \% \mathrm{Cl}$ & $p$-value ${ }^{*}$ \\
\hline Age ( $\geq 20$ vs. $<20$ yr) & 1.05 & $0.29-3.81$ & 0.946 & 1.41 & $0.41-4.83$ & 0.581 \\
\hline Sex (male vs. female) & 0.57 & $0.17-1.92$ & 0.365 & 1.51 & $0.47-4.89$ & 0.492 \\
\hline \multicolumn{7}{|l|}{ Disease } \\
\hline ALL & 1 (reference) & - & - & 1 (reference) & - & - \\
\hline $\mathrm{AML}$ & 0.82 & $0.13-5.08$ & 0.832 & 1.64 & $0.29-9.29$ & 0.574 \\
\hline Other & NA & NA & NA & 1.64 & $0.29-9.29$ & 0.574 \\
\hline Total dose (12 vs. 8-9.6 Gy & 0.22 & $0.02-2.62$ & 0.230 & NA & NA & NA \\
\hline \multicolumn{7}{|l|}{ Dose rate (cGy/min) } \\
\hline $5-9.99$ & 1 (reference) & - & - & 1 (reference) & - & - \\
\hline $10-14.99$ & 1.63 & $0.41-6.46$ & 0.487 & 0.44 & $0.10-1.93$ & 0.276 \\
\hline$\geq 15$ & 0.28 & $0.05-1.69$ & 0.166 & 2.14 & $0.52-8.81$ & 0.291 \\
\hline \multicolumn{7}{|l|}{ Donor type } \\
\hline Matched sibling & 1 (reference) & - & - & 1 (reference) & - & - \\
\hline Matched unrelated & 1.11 & $0.24-5.07$ & 0.892 & 12.67 & $1.46-110.30$ & 0.021 \\
\hline Mismatched related & 8.00 & $0.58-110.27$ & 0.120 & NA & NA & NA \\
\hline \multicolumn{7}{|l|}{ Cell type } \\
\hline Bone marrow & 1 (reference) & - & - & 1 (reference) & - & - \\
\hline Peripheral blood & 0.56 & $0.15-2.03$ & 0.376 & 4.09 & $1.14-14.66$ & 0.031 \\
\hline Cord blood & NA & NA & NA & NA & NA & NA \\
\hline \multicolumn{7}{|l|}{ Conditioning regimen } \\
\hline Cy & 1 (reference) & - & - & 1 (reference) & - & - \\
\hline $\mathrm{Cy}+$ other & 7.20 & $1.24-41.94$ & 0.028 & 0.48 & $0.08-2.71$ & 0.403 \\
\hline Other & NA & NA & NA & 1.91 & $0.37-9.87$ & 0.443 \\
\hline Acute GVHD prophylaxis (yes MTV vs. no MTX) & 0.29 & $0.08-1.08$ & 0.064 & 2.98 & $0.70-12.67$ & 0.139 \\
\hline Smoking history (yes vs. no) & 1.03 & $0.09-12.32$ & 0.979 & 3.11 & $0.26-36.87$ & 0.368 \\
\hline Pre-transplant pulmonary comorbidity (yes vs. no) & 1.04 & $0.17-6.35$ & 0.970 & 0.69 & $0.12-4.21$ & 0.692 \\
\hline \multicolumn{7}{|l|}{ Acute GVHD } \\
\hline No & 1 (reference) & - & - & 1 (reference) & - & - \\
\hline Grade 1-2 & 0.63 & $0.17-2.32$ & 0.483 & 1.41 & $0.39-5.13$ & 0.602 \\
\hline Grade 3-4 & 0.41 & $0.07-2.58$ & 0.341 & 1.47 & $0.28-7.63$ & 0.649 \\
\hline \multicolumn{7}{|l|}{ CR group } \\
\hline CR1 & 1 (reference) & - & - & 1 (reference) & - & - \\
\hline$\geq \mathrm{CR} 2$ & 1.07 & $0.29-3.92$ & 0.923 & 1.78 & $0.50-6.37$ & 0.377 \\
\hline Refractory & 1.78 & $0.10-31.98$ & 0.696 & NA & NA & NA \\
\hline PreFEV1 (per \% increase) & 0.98 & $0.93-1.03$ & 0.327 & 1.03 & $0.99-1.08$ & 0.129 \\
\hline PreFVC (per \% increase) & 0.96 & $0.90-1.01$ & 0.111 & 1.02 & $0.98-1.07$ & 0.243 \\
\hline PreDLCO (per \% increase) & 1.36 & $0.89-2.06$ & 0.151 & 0.90 & $0.79-1.03$ & 0.129 \\
\hline
\end{tabular}

$\mathrm{OR}$, odds ratio; $\mathrm{Cl}$, confidence interval; $\mathrm{NA}$, not applicable; $\mathrm{ALL}$, acute lymphoblastic leukemia; $\mathrm{AML}$, acute myeloid leukemia; $\mathrm{Cy}$, $\mathrm{Cyclo-}$ phosphamide; GVHD, graft-versus-host disease; MTX, methotrexate; CR, complete response; FEV1, forced expiratory volume in 1 second; FVC, forced vital capacity; DLCO, diffusion capacity of carbon monoxide.

${ }^{*} p$-values in bold-type represent those that reached statistical significance.

not have much impact on pulmonary toxicity $[27,28]$. Other radiation-related factors such as total dose, dose per fraction, and radiation therapy techniques have remained the same, which makes comparing the effect of the dose rate between patients of different eras more amenable. In the present study, our data demonstrated that the dose rate did not significantly affect pulmonary toxicity (Table 3), which is consistent with observations is a previous study [27]. Although our sample size was not large enough to draw a definitive conclusion regarding this issue, risk stratification based on radiation-related and other clinical factors, such as conditioning intensity and donor type, are necessary to identify the subset of patients who are likely to develop pulmonary toxicity after stem cell transplantation. The importance of considering multiple clinical 
Table 4. Multivariate analysis of factors associated with infectious pneumonia and idiopathic pneumonia syndrome

\begin{tabular}{|c|c|c|c|c|c|c|}
\hline \multirow{2}{*}{ Variable } & \multicolumn{3}{|c|}{ Infectious pneumonia } & \multicolumn{3}{|c|}{ Idiopathic pneumonia syndrome } \\
\hline & $\mathrm{OR}$ & $95 \% \mathrm{Cl}$ & $p$-value ${ }^{*}$ & $\mathrm{OR}$ & $95 \% \mathrm{Cl}$ & $p$-value ${ }^{*}$ \\
\hline \multicolumn{7}{|l|}{ Donor type } \\
\hline Matched sibling & & & & 1 (reference) & - & - \\
\hline Matched unrelated & & & & 12.67 & $1.46-110.30$ & 0.021 \\
\hline Mismatched related & & & & NA & NA & NA \\
\hline \multicolumn{7}{|l|}{ Cell type } \\
\hline Bone marrow & 1 (reference) & - & - & & & \\
\hline Peripheral blood & 0.5 & $0.12-2.14$ & 0.352 & & & \\
\hline Cord blood & NA & NA & NA & & & \\
\hline \multicolumn{7}{|l|}{ Conditioning regimen } \\
\hline Cy & 1 (reference) & - & - & & & \\
\hline $\mathrm{Cy}+$ other & 6.19 & $0.90-42.56$ & 0.064 & & & \\
\hline Other & NA & NA & NA & & & \\
\hline
\end{tabular}

OR, odds ratio; $\mathrm{Cl}$, confidence interval; NA, not applicable; Cy, cyclophosphamide; GVHD, graft-versus-host disease; MTX, methotrexate. ${ }^{*} p$-values in bold-type represent those that reached statistical significance.

factors in HSCT was emphasized in a previous investigation in which patients were categorized into subgroups based on combinations of risk factors, including intensity of the conditioning regimen, transplanted cell type, donor type, and whether TBI was applied. In that study, treatment outcomes and toxicities varied in the different subgroups [29].

In contrast to IP (which significantly affected mortality), we found that IPS has less of an effect on TRM and OS (Fig. 2). This finding was consistent with that of a recent study that showed that IP, but not IPS, had a detrimental effect on TRM and OS rates [10]. However, the importance of IPS should not be discounted, as it contributed considerably to TRM; moreover, noninfectious lung toxicity is still reported as a contributing cause of death in patients.

In the current study, patients were classified as having pulmonary toxicities when they had both clinical symptoms and objective evidences on radiographic or pulmonary function tests. Although patients could have presented with asymptomatic pneumonia, our routine image follow-up consisted of plain radiography only, which makes detection of asymptomatic pneumonia difficult. Chest computed tomography scans were additionally performed when pulmonary symptoms were observed. Of 22 patients who were classified as not developing IPS or IP, no patient had radiologic evidence of pneumonia on plain radiography. This may be because mild pulmonary toxicities cannot be easily detected through plain radiography.

Our study has several limitations that are inherent to its retrospective nature. The sample size was too small to conduct a complete statistical analysis. There was a possibility of falsenegative results with respect to pathogen detection, although we examined patient specimens using well-established techniques to identify the cause of IP. It is also possible that the infectious and non-infectious etiologies could overlap, so IP might be obscured by pathogen discovery because we stratified patients based on the presence or absence of concurrent infection dichotomically. However, because similar approaches have been used in previous studies, they appear reasonable for distinguishing IPS from IP, given the definition of IPS $[7,10]$.

In conclusion, our data support our hypothesis that IP and IPS have distinct risk factors as well as different inherent pathogeneses. Our findings demonstrate that multiple conditioning regimens may potentially cause patients to be at a high risk of developing IP. Patients who received stem cells from an unrelated donor had a higher risk of developing IPS than those who received stem cells from a matched sibling donor. Identifying patients with risk factors for IP and IPS before complications develop can lead to appropriate preventive strategies and work-ups for early recognition of these diseases, which may further reduce mortality due to pulmonary complications.

\section{Conflict of Interest}

No potential conflict of interest relevant to this article was reported. 


\section{Acknowledgments}

This study was supported by a 2017 research seed-money grant for new faculty from Yonsei University College of Medicine (2017-32-0029).

\section{References}

1. Hartman AR, Williams SF, Dillon JJ. Survival, diseasefree survival and adverse effects of conditioning for allogeneic bone marrow transplantation with busulfan/ cyclophosphamide vs total body irradiation: a meta-analysis. Bone Marrow Transplant 1998;22:439-43.

2. Socie G, Clift RA, Blaise D, et al. Busulfan plus cyclophosphamide compared with total-body irradiation plus cyclophosphamide before marrow transplantation for myeloid leukemia: long-term follow-up of 4 randomized studies. Blood 2001;98:3569-74.

3. Chaillet MP, Cosset JM, Socie $G$, et al. Prospective study of the clinical symptoms of therapeutic whole body irradiation. Health Phys 1993;64:370-4.

4. Ozsahin M, Pene F, Cosset JM, Laugier A. Morbidity after total body irradiation. Semin Radiat Oncol 1994;4:95-102.

5. Neiman PE, Reeves W, Ray G, et al. A prospective analysis interstitial pneumonia and opportunistic viral infection among recipients of allogeneic bone marrow grafts. J Infect Dis 1977;136:754-67.

6. Thomas ED, Buckner CD, Banaji M, et al. One hundred patients with acute leukemia treated by chemotherapy, total body irradiation, and allogeneic marrow transplantation. Blood 1977:49:511-33.

7. Clark JG, Hansen JA, Hertz MI, Parkman R, Jensen L, Peavy HH. NHLBI workshop summary. Idiopathic pneumonia syndrome after bone marrow transplantation. Am Rev Respir Dis 1993;147(6 Pt 1):1601-6.

8. Morgan TL, Falk PM, Kogut N, Shah KH, Tome M, Kagan AR. A comparison of single-dose and fractionated total-body irradiation on the development of pneumonitis following bone marrow transplantation. Int J Radiat Oncol Biol Phys 1996;36:61-6.

9. Kelsey $C R$, Horwitz ME, Chino JP, et al. Severe pulmonary toxicity after myeloablative conditioning using total body irradiation: an assessment of risk factors. Int J Radiat Oncol Biol Phys 2011;81:812-8.

10. Abugideiri $M, N a n d a ~ R H, B u t k e r ~ C$, et al. Factors influencing pulmonary toxicity in children undergoing allogeneic hematopoietic stem cell transplantation in the setting of total body irradiation-based myeloablative conditioning. Int J
Radiat Oncol Biol Phys 2016;94:349-59.

11. Savani BN, Montero A, Wu C, et al. Prediction and prevention of transplant-related mortality from pulmonary causes after total body irradiation and allogeneic stem cell transplantation. Biol Blood Marrow Transplant 2005;11:223-30.

12. Ethier MC, Blanco $E$, Lehrnbecher T, Sung L. Lack of clarity in the definition of treatment-related mortality: pediatric acute leukemia and adult acute promyelocytic leukemia as examples. Blood 2011;118:5080-3.

13. Park J, Choi EK, Kim JH, et al. Effects of total body irradiationbased conditioning on allogeneic stem cell transplantation for pediatric acute leukemia: a single-institution study. Radiat Oncol J 2014;32:198-207.

14. Glucksberg H, Storb R, Fefer A, et al. Clinical manifestations of graft-versus-host disease in human recipients of marrow from HL-A-matched sibling donors. Transplantation 1974;18:295304.

15. Fielding AK, Rowe JM, Richards SM, et al. Prospective outcome data on 267 unselected adult patients with Philadelphia chromosome-positive acute lymphoblastic leukemia confirms superiority of allogeneic transplantation over chemotherapy in the pre-imatinib era: results from the International ALL Trial MRC UKALLXII/ECOG2993. Blood 2009;113:4489-96.

16. Ho VT, Weller E, Lee SJ, Alyea EP, Antin JH, Soiffer RJ. Prognostic factors for early severe pulmonary complications after hematopoietic stem cell transplantation. Biol Blood Marrow Transplant 2001;7:223-9.

17. Chen $C S$, Boeckh $M$, Seidel $K$, et al. Incidence, risk factors, and mortality from pneumonia developing late after hematopoietic stem cell transplantation. Bone Marrow Transplant 2003;32:515-22.

18. Pino y Torres JL, Bross DS, Lam WC, Wharam MD, Santos GW, Order SE. Risk factors in interstitial pneumonitis following allogenic bone marrow transplantation. Int J Radiat Oncol Biol Phys 1982;8:1301-7.

19. Carruthers SA, Wallington MM. Total body irradiation and pneumonitis risk: a review of outcomes. $\mathrm{Br} J$ Cancer 2004:90:2080-4.

20. Zhang MJ, Davies SM, Camitta BM, et al. Comparison of outcomes after HLA-matched sibling and unrelated donor transplantation for children with high-risk acute lymphoblastic leukemia. Biol Blood Marrow Transplant 2012;18:1204-10.

21. Toruner M, Loftus EV Jr, Harmsen WS, et al. Risk factors for opportunistic infections in patients with inflammatory bowe disease. Gastroenterology 2008;134:929-36.

22. Mohty $M$, Jacot $W$, Faucher $C$, et al. Infectious complications following allogeneic HLA-identical sibling transplantation with 
antithymocyte globulin-based reduced intensity preparative regimen. Leukemia 2003;17:2168-77.

23. Martino R, Caballero MD, Canals $C$, et al. Reduced-intensity conditioning reduces the risk of severe infections after allogeneic peripheral blood stem cell transplantation. Bone Marrow Transplant 2001;28:341-7.

24. Hamadani M, Blum W, Phillips G, et al. Improved nonrelapse mortality and infection rate with lower dose of antithymocyte globulin in patients undergoing reduced-intensity conditioning allogeneic transplantation for hematologic malignancies. Biol Blood Marrow Transplant 2009;15:1422-30.

25. Wolden SL, Rabinovitch RA, Bittner NH, et al. American College of Radiology (ACR) and American Society for Radiation Oncology (ASTRO) practice guideline for the performance of total body irradiation (TBI). Am J Clin Oncol 2013;36:97-101.
26. Belkacemi Y, Pene F, Touboul E, et al. Total-body irradiation before bone marrow transplantation for acute leukemia in first or second complete remission. Results and prognostic factors in 326 consecutive patients. Strahlenther Onkol 1998;174:92-104.

27. Girinsky T, Benhamou E, Bourhis JH, et al. Prospective randomized comparison of single-dose versus hyperfractionated total-body irradiation in patients with hematologic malignancies. J Clin Oncol 2000;18:981-6.

28. Ozsahin M, Pene F, Touboul E, et al. Total-body irradiation before bone marrow transplantation: results of two randomized instantaneous dose rates in 157 patients. Cancer 1992;69:2853-65.

29. Jagasia $M$, Arora $M$, Flowers $M E$, et al. Risk factors for acute GVHD and survival after hematopoietic cell transplantation. Blood 2012;119:296-307. 